\title{
A SINGULAR FINITE ELEMENT FOR STOKES FLOW: THE STICK-SLIP PROBLEM
}

\author{
GEORGIOS C. GEORGIOU,* LORRAINE G. OLSON, WILLIAM W. SCHULTZ AND \\ SUSAN SAGAN \\ Department of Mechanical Engineering and Applied Mechanics (*Department of Chemical Engineering), The University \\ of Michigan, Ann Arbor, MI 48109, U.S.A.
}

\begin{abstract}
SUMMARY
Abrupt changes in boundary conditions in viscous flow problems give rise to stress singularities. Ordinary finite element methods account effectively for the global solution but perform poorly near the singularity. In this paper we develop singular finite elements, similar in principle to the crack tip elements used in fracture mechanics, to improve the solution accuracy in the vicinity of the singular point and to speed up the rate of convergence. These special elements surround the singular point, and the corresponding field shape functions embody the form of the singularity. Because the pressure is singular, there is no pressure node at the singular point. The method performs well when applied to the stick-slip problem and gives more accurate results than those from refined ordinary finite element meshes.
\end{abstract}

KEY wORDS Singular finite elements Stokes flow Stress singularity

\section{INTRODUCTION}

The extrusion of a viscous jet from a die into an inviscid medium has been the focus of a plethora of theoretical, experimental and computational works because of its importance in polymerprocessing operations and other industrial applications. Two characteristics of the extrudate-swell problem are the expansion of the jet at low Reynolds numbers and the presence of a stress singularity at the exit of the die. The problem is analytically intractable because of the nonlinear boundary conditions on the free surface. Numerical methods, especially finite elements, have been used extensively to overcome this difficulty. ${ }^{1-4}$

The stress singularity, which is the focus of this work, arises from the abrupt change in the boundary condition at the exit of the die; its analysis is very important for a good comprehension of the extrudate-swell phenomenon. ${ }^{5}$ Generally speaking, singularities require special treatment in the numerical solution of singular problems. In finite element analysis, local refinement around the singular point is often employed in order to improve the accuracy. However, the accuracy and the rate of convergence are not in general satisfactory. Standard finite elements predict inaccurate stresses around the lip of the die; the stresses cannot be infinite at the singular point as the local asymptotic solution demands. Furthermore, the stiffness matrix becomes large as the mesh is refined and therefore the computational cost increases. Silliman and Scriven ${ }^{6}$ applied a slip boundary condition in the upstream vicinity of the contact line to alleviate the stress singularity, since the no-slip boundary condition may be not valid in this region. They do not adequately resolve the singularity as the slip coefficient goes to zero, but they determine that a wide range of slip coefficients give the same global behaviour.

0271-2091/89/111353-15\$07.50

(C) 1989 by John Wiley \& Sons, Ltd.

Received 29 July 1988

Revised 9 December 1988 
Incorporating the nature of the singularity, obtained by an asymptotic analysis, in the numerical solution proves to be a very effective way to improve the accuracy in the neighbourhood of the singularity and to speed up the rate of convergence. This idea has been successfully adopted in solving mainly fracture mechanics problems by a variety of methods: finite differences, ${ }^{7}$ finite elements,,${ }^{89}$ boundary and global elements ${ }^{10,11}$ and spectral methods. ${ }^{12}$ Two singular finite element approaches appear in the literature [11]:

1. Singular basis function approach. A set of supplementary basis functions chosen to reproduce the leading terms of the singularity solution is added to the standard finite element solution expansion. ${ }^{8,13}$ The singular functions are usually defined over several elements and can span the entire domain.

2. Singular element approach. Special elements are used in a small region around the singularity, while conventional elements are used in the rest of the domain. The various proposed elements can be classified in three categories:

(a) Embodied singularity elements-Special elements are employed around the singular point, and the corresponding field shape functions embody the form of the singularity. $9,14,15$

(b) Embedded singularity elements - The leading terms of the singularity expansion are used to describe the full solution over a multinode element surrounding the singular point, and conventional elements are used elsewhere. ${ }^{16}$

(c) Singular isoparametric elements-Singular geometric transformations, defined on the elements surrounding the singular point, can provide finite element approximations with the desired singular behaviour. The transformation becomes singular by properly changing the position of the midnodes, e.g. quarter-point elements. ${ }^{17-19}$

There is a wealth of published work on singular finite element approaches owing to their success in fracture mechanics. A review is given by Gallagher ${ }^{20}$ in which the various approaches are critically examined and their relative advantages and disadvantages are discussed.

The main objective of the present work is to use the ideas developed in solid mechanics to solve singular problems in fluid mechanics, which are more complicated because, in addition to the velocity derivatives, the pressure-a primary unknown-is also singular. Another complication arises in free surface problems in which we have curved boundaries and the form of the singularity is not exactly known.

We have chosen to solve the stick-slip problem at this stage, using embodied singularity elements. The stick-slip problem is a limiting case of the extrudate-swell problem, equivalent to the creeping Newtonian planar jet at infinite surface tension; no expansion occurs in this case and the free surface is flat. The boundary conditions change suddenly from no slip along the wall to perfect slip along the planar 'free' surface. For this particular case, the non-linearity due to the free surface is eliminated and the stick-slip problem is amenable to analytical solution. The planar problem was solved analytically by Richardson ${ }^{21}$ using the Wiener-Hopf method, and by Sturges $^{22}$ using the eigenfunction method. Nevertheless, the local solution near the lip is just a special case of the general flow of a viscous fluid near a corner formed by a solid wall and a flat free surface; the local analysis is described by Michael $^{23}$ and Moffat ${ }^{24}$ The velocity components vary as the square root of the radial distance from the singular point, resulting in an inverse square root singularity for the stresses.

The singular elements developed here are similar to those introduced by Tracey ${ }^{9}$ for the crack tip problem, which also exhibits an inverse square root singularity for stresses. Compared to the singular basis function approach or the embedded singularity elements, the embodied singularity elements can be included more easily in a general finite element code and do not require 
knowledge of the angular dependence of the solution. Furthermore, they are compatible with the adjoining ordinary elements and can be extended to free surface flow problems, e.g. the extrudate-swell problem.

Even though the singular isoparametric elements have been successful in dealing with crack problems in fracture mechanics ${ }^{17,19}$ and are equivalent to the embodied singularity elements in some cases, the latter are more appropriate for fluid flow problems for the following reasons. (a) Singular isoparametric elements cannot handle singular primary variables. The proposed elements can be constructed with no node at the singular point for the singular primary variables. (b) Singular isoparametric elements with curved sides are difficult to use without loss of accuracy. Consequently, they are not as effective as the embodied singularity elements for free surface problems. (c) In order to describe general power-type singularities with isoparametric elements, one has either to increase the number of nodes per element or to construct special ficld shape functions which in combination with the distortion of the physical element will yield the desired behaviour. With the embodied singularity elements one has to modify only the field shape functions.

Singular elements can be applied to a general class of singular fluid mechanics problems, provided that the radial form of the local solution is known or can be obtained by an asymptotic analysis. Thus singular finite elements can be used to solve non-zero Reynolds number or nonNewtonian flows. For a non-zero Reynolds number the analysis is valid near the singularity, where the viscous effect is dominant, and the form of the local solution remains the same. Schultz and Gervasio ${ }^{25}$ showed that the resulting local eigenproblem can be solved if the slip surface is flat or the curvature of the free surface is integrable. An asymptotic analysis to obtain the radial form of the singularity is also possible for various viscoelastic flows such as the stick-slip flow of an Oldroyd-B or a second-order fluid. ${ }^{26,27}$ Apelian $e t$ al., ${ }^{27}$ in solving the stick-slip problem with a modified upper-convected Maxwell model, showed that the elastic contribution to the stress varies as $r^{-1 / 5}$. The developed elements can easily embody both the Newtonian and the elastic contributions by appropriately modifying the field shape functions. ${ }^{28}$ Therefore the method can be used in viscoelastic flows provided that the encountered stresses are integrable.

As we will see in the following sections, the embodied singularity elements give results that are more accurate than those from conventional finite elements even if coarser meshes are used. The governing equations and the asymptotic solution are given in Section 2 . The finite element formulation, the construction of the field shape functions and the numerical integration over the singular elements are discussed in Section 3. Finally, the results are presented and discussed in Section 4.

\section{GOVERNING EQUATIONS AND LOCAL SOLUTION}

The geometry, governing equations and boundary conditions for the stick-slip problem are depicted in Figure 1. The flow is governed by the Stokes equation and continuity:

$$
\nabla \cdot \mathbf{T}=0
$$

and

$$
\nabla \cdot \mathbf{V}=\mathbf{0}
$$

Here $\mathrm{T}$ is the stress tensor for a Newtonian liquid, measured in units of $\mu U / H, \mu$ is the viscosity, $U$ is the mean velocity in the channel and $H$ is the channel half-width. The velocity $\mathbf{V}$ is scaled by $U$.

The form of the singularity is known as a special case of the steady plane flow near a corner of angle $\omega$ formed by a rigid boundary and a flat free surface, as shown in Figure 1(b). This general 


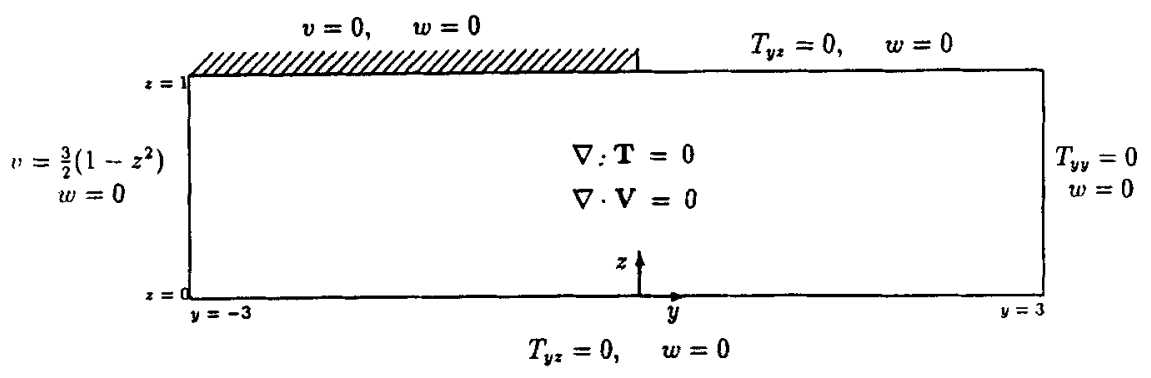

(a)

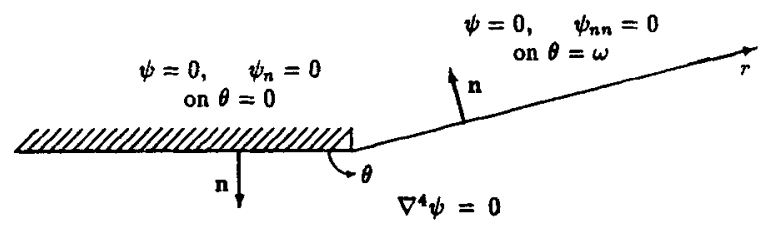

(b)

Figure 1. (a) The stick-slip problem. (b) Local analysis of the singularity

flow was analysed in plane polar co-ordinates $(r, \theta)$ by Michael ${ }^{23}$ and Moffat. ${ }^{24}$ For the stick-slip case, $\omega=\pi$ and there are two possible sets of solutions for the streamfunction $\psi$ :

$$
\psi=r^{\lambda+1} \alpha_{\lambda}[\cos (\lambda+1) \theta-\cos (\lambda-1) \theta] \text { for } \lambda=\frac{1}{2}, \frac{3}{2}, \frac{5}{2}, \ldots
$$

and

$$
\psi=r^{\lambda+1} \beta_{\lambda}[(\lambda-1) \sin (\lambda+1) \theta-(\lambda+1) \sin (\lambda-1) \theta], \text { for } . \lambda=2,3,4, \ldots,
$$

where $\alpha_{\lambda}$ and $\beta_{\lambda}$ are arbitrary constants. The velocity components are proportional to $r^{\lambda}$. For $\theta=\pi$ the contributions of (4) become zero and the $y$-component of velocity is given by

$$
v=2 \alpha_{1 / 2} y^{1 / 2}-2 \alpha_{3 / 2} y^{3 / 2}+2 \alpha_{5 / 2} y^{5 / 2}+O\left(y^{7 / 2}\right) \text {. }
$$

From the analytical solution the first constant is $\alpha_{1 / 2}=\sqrt{ }(3 / 2 \pi)=0.690988$. As indicated by Ingham and Kelmanson, ${ }^{10}$ the value $\alpha_{1 / 2}=0.581$ given by Richardson ${ }^{21}$ is wrong. Pressure and stresses are proportional to $r^{\lambda-1}$, and the inverse square root singularity is due to the first term of (3). The normal stress $T_{n n}$ on the slip surface is non-singular and represents a very severe test for the numerical calculations. It turns out that only the pressure contributions from the integer-power solutions are non-zero and the normal stress on the slip surface is of the form

$$
\mathrm{T}_{\mathrm{nn}}=c-24 \beta_{2} y+48 \beta_{3} y^{2}+80 \beta_{4} y^{3}+O\left(y^{4}\right)
$$

where $c$ is a constant. 


\section{FINITE ELEMENT FORMULATION}

The finite element method is well established ${ }^{29,30}$ and therefore we emphasize only the aspects related to singular finite elements: construction of field shape functions and numerical integration over singular elements.

The physical domain of the stick-slip problem, shown in Figure 1, extends three channel halfwidths upstream and downstream, a distance sufficiently large to assure the validity of the imposed boundary conditions. Taking the outflow and inflow planes farther from the lip causes negligible changes in the predicted flow field.

In the finite element discretization we use singular triangular-shaped elements in a small core around the singularity and ordinary rectangular elements in the rest of the domain, as illustrated in Figures 2-4. In the ordinary elements, the basis functions are biquadratic for the velocities and bilinear for the pressure. These elements are mapped on a $2 \times 2$ master element in $(s, t)$ coordinates with biquadratic shape functions.

The construction of field shape functions, describing derivative singularities of the general form $r^{n-1}(0<n<1)$, has been the central subject of various works. ${ }^{28,31}$ Hughes and Akin ${ }^{28}$ presented an algorithm for generating shape functions from an arbitrary starting set of independent functions. The generated functions are capable of representing different singular behaviours within the element. For the present problem, a simple Lagrange interpolation in terms of $\sqrt{ } r$ is adequate for deriving the field shape functions. Figure 2 shows the rectangular master element with the $(s, t)$ co-ordinates mapped approximately to the physical radial and circumferential coordinates respectively ${ }^{9}$ (see Appendix). The field shape functions must embody the singularity and be compatible with the adjacent ordinary elements. Hence the shape functions for the velocities $\Phi^{i}$ are of the general form

$$
\Phi^{i}=N^{i}(s) P^{i}(t)
$$

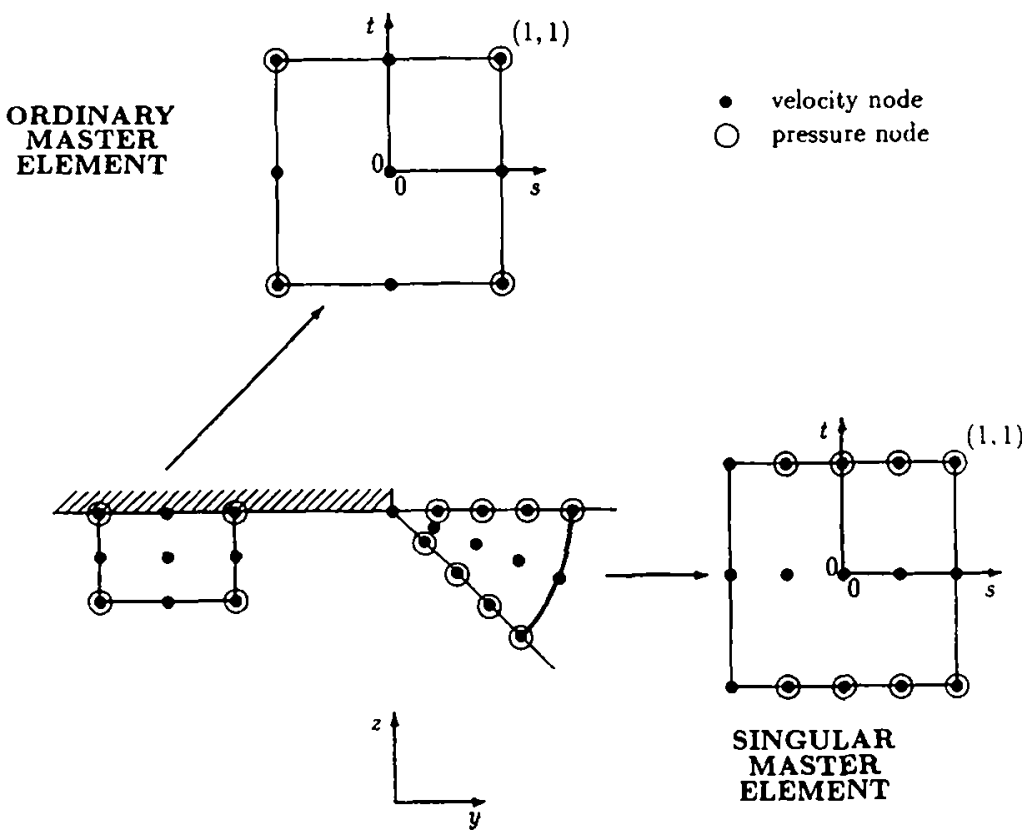

Figure 2. Ordinary and singular elements 


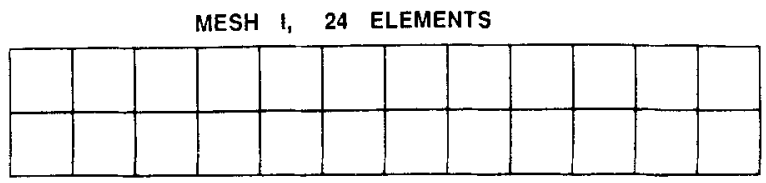

MESH II, 96 ELEMENTS

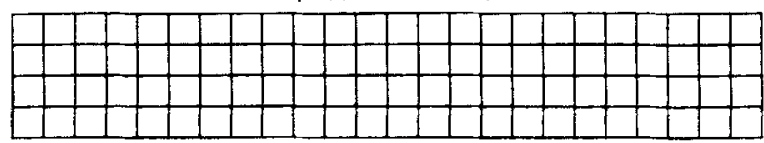

MESH III, 384 ELEMENTS

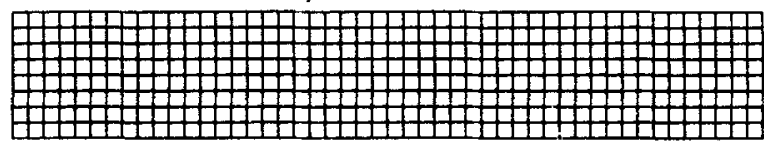

MESH IV, 450 ELEMENTS

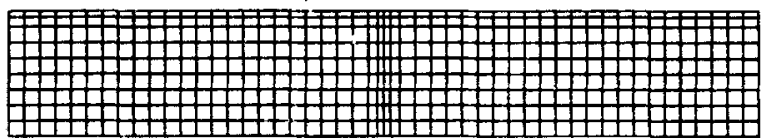

MESH V, 520 ELEMENTS

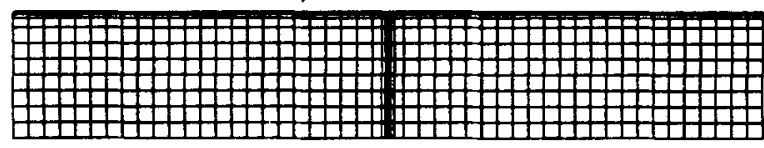

Figure 3. Ordinary element meshes

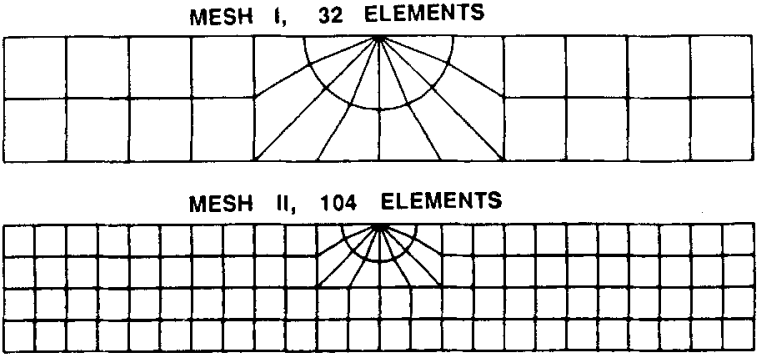

MESH III, 392 ELEMENTS

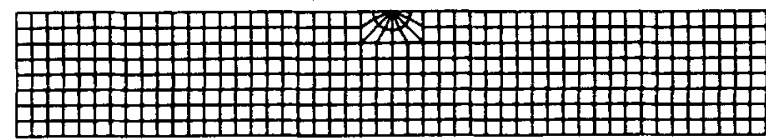

Figure 4. Singular element meshes

with $P$ varying quadratically with $t$ to maintain compatibility with the adjoining elements. Three velocity nodes are thus needed in the $t$-direction. At $s=-1, P=1$ and the three velocity nodes collapse to one node with only two degrees of freedom for the two velocity components. To include the first two contributions from both (3) and (4), five velocity nodes are needed in the $s$-direction and $N^{i}$ takes the form

$$
N^{i}(s)=a+b(1+s)^{1 / 2}+c(1+s)+d(1+s)^{3 / 2}+e(1+s)^{2} .
$$


Requiring that $N^{i}\left(s_{j}\right)=\delta_{i j}$ and using Lagrange interpolation with respect to the square root of the radial co-ordinate gives

$$
\begin{aligned}
& N^{1}=(1-\sqrt{ } x)\left[1-\sqrt{ }\left(\frac{4}{3} x\right)\right][1-\sqrt{ }(2 x)][1-\sqrt{ }(4 x)],
\end{aligned}
$$

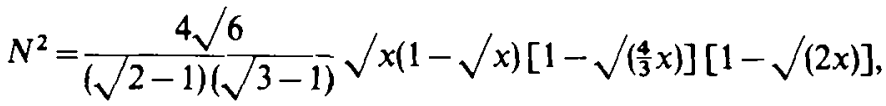

$$
\begin{aligned}
& N^{3}=-\frac{2 \sqrt{3}}{(\sqrt{3-\sqrt{2})(3-2 \sqrt{2})}} \sqrt{ } x(1-\sqrt{ } x)\left[1-\sqrt{ }\left(\frac{4}{3} x\right)\right][1-\sqrt{ }(4 x)] \text {, } \\
& N^{4}=-\frac{4 \sqrt{2}}{\sqrt{3}(2-\sqrt{3})(\sqrt{3}-\sqrt{2})(\sqrt{3-1})} \sqrt{ } x(1-\sqrt{ } x)[1-\sqrt{ }(2 x)][1-\sqrt{ }(4 x)] \text {, } \\
& N^{5}=-\frac{\sqrt{3}}{(2-\sqrt{3})(\sqrt{2}-1)} \sqrt{ } x\left[1-\sqrt{\left(\frac{4}{3} x\right)}\right][1-\sqrt{ }(2 x)][1-\sqrt{ }(4 x)],
\end{aligned}
$$

where $x=(1+s) / 2$ and the nodes are numbered sequentially in the $s$-direction.

For the pressure shape functions $\Psi^{i}$ we use a lower-order representation:

$$
\Psi^{i}=M^{i}(s) Q^{i}(t)
$$

with $Q$ varying linearly with $t$ (two pressure nodes in $t$-direction) and $M^{i}$ having the same form as $\mathrm{d} N^{i} / \mathrm{d} s$ :

$$
M^{i}(s)=a^{\prime}(1+s)^{-1 / 2}+b^{\prime}+c^{\prime}(1+s)^{1 / 2}+d^{\prime}(1+s) .
$$

Four pressure nodes are needed in the $s$-direction and because pressure is singular no node is placed at $s=-1$ (Figure 2). Using Lagrange interpolation gives

$$
\begin{aligned}
& M^{1}=\frac{\sqrt{ } 6}{(\sqrt{3}-1)(\sqrt{2-1})} \frac{1}{\sqrt{x}}(1-\sqrt{ } x)\left[1-\sqrt{ }\left(\frac{4}{3} x\right)\right][1-\sqrt{ }(2 x)], \\
& M^{2}=-\frac{\sqrt{3}}{(3-2 \sqrt{2})(\sqrt{3}-\sqrt{2})} \frac{1}{\sqrt{x}}(1-\sqrt{ } x)\left[1-\sqrt{ }\left(\frac{4}{3} x\right)\right][1-\sqrt{ }(4 x)], \\
& M^{3}=\frac{\sqrt{ } 6}{(2-\sqrt{3})(\sqrt{3}-\sqrt{2})(\sqrt{3}-1)} \frac{1}{\sqrt{x}}(1-\sqrt{ } x)[1-\sqrt{ }(2 x)][1-\sqrt{ }(4 x)], \\
& M^{4}=-\frac{\sqrt{ } 3}{(2-\sqrt{3})(\sqrt{2}-1)} \frac{1}{\sqrt{x}}\left[1-\sqrt{ }\left(\frac{4}{3} x\right)\right][1-\sqrt{ }(2 x)][1-\sqrt{ }(4 x)],
\end{aligned}
$$

with the nodes numbered sequentially in the $s$-direction. Note that the constructed shape functions satisfy the conditions $\Sigma \Phi^{i}=1, \Sigma \Psi^{i}=1$ and are linearly independent. The master element is mapped to the triangular physical element by means of ordinary polynomial shape functions of the fourth order in the $s$-direction and the second order in the $t$-direction.

Applying the Galerkin principle, we weight the momentum and continuity equations by the velocity and pressure basis functions. After applying Green's theorem we have

$$
\int_{\boldsymbol{S}} \mathbf{n} \cdot \mathbf{T} \Phi^{i} \mathrm{~d} S-\int_{\Omega} \mathbf{T} \cdot \nabla \Phi^{i} \mathrm{~d} \Omega=0, \quad i=1,2, \ldots, N_{u},
$$

and

$$
\int_{\Omega} \nabla \cdot \mathrm{V} \Psi^{i} \mathrm{~d} \Omega=0, \quad i=1,2, \ldots, N_{p}
$$


where $\Omega$ and $S$ are the domain and its boundary, $n$ is the unit normal vector pointing outward from the boundary, and $N_{u}$ and $N_{p}$ are the numbers of velocity and pressure nodes respectively. The total number of unknowns is $2 N_{u}+N_{p}$. Equations (11) and (12) consist of a system of linear equations efficiently solved by standard subroutines, e.g. frontal methods.

Standard $3 \times 3$ Gaussian quadrature is used for the integration over the ordinary elements. As noted by various investigators, ${ }^{14,32,33}$ a standard quadrature rule of low order is inappropriate for the integration over the singular elements. Special quadrature rules for singular elements with $r^{n}$ behaviour, with the quadrature points and weights varying with the exponent $n$, are described by Solecki and Swedlow. ${ }^{32}$ However, for the square root behaviour examined here, the substitution

$$
\sqrt{ }(1+s)=\frac{\xi+1}{\sqrt{2}}
$$

transforms the encountered integrands into simple polynomials in the $\xi$-domain, and therefore standard Gauss-Legendre quadrature suffices for an exact integration. This treatment is equivalent to modifying the standard Gauss weights $w_{k}^{\mathrm{G}}$ and points $\gamma_{i}^{\mathrm{G}}$ as follows:

$$
\gamma_{i}=\frac{\left(\gamma_{i}^{\mathrm{G}}+1\right)^{2}}{2}-1
$$

and

$$
w_{i}=w_{i}^{G}\left(\gamma_{i}^{G}+1\right)
$$

for the integration along the radial direction. We use a $5 \times 3$ modified Gaussian quadrature for integration over the singular elements.

\section{RESULTS AND DISCUSSION}

The stick-slip problem was solved using both ordinary and singular finite elements. Five ordinary meshes and three singular meshes, shown in Figures 3 and 4, were constructed for this purpose. The first three ordinary meshes (I, II, III) were uniform and consisted of 24,96 and 384 square elements. The last two meshes (IV, V) were obtained by refining only the row and columns around the tip; they consisted of 450 and 520 elements. To generate the singular meshes, we modified the uniform ordinary meshes: eight ordinary square elements were replaced by eight singular triangular-shaped elements and eight ordinary transition elements in the circular pattern shown in Figure 4. Of course, uniform meshes are by no means optimum for the stick-slip problem, but in this work they appear to be more appropriate for comparisons between singular and ordinary finite elements.

The solution of the stick-slip problem obtained with the finest singular mesh is illustrated in Figure 5, where we plot the velocity and pressure contours and the streamlines. The results clearly depict the rearrangement of the flow from a Poiseuille flow to a uniform flow regime.

The singular and ordinary finite element results far from the singular point are in good agreement. Both methods predict essentially the same centreline pressure (Figure 6); it varies linearly with the axial distance inside the die and goes smoothly to zero in the jet. However, the results around the singularity differ dramatically. We focus on two important quantities: the normal stress along the wall and the slip surface, and the velocity along the slip surface.

The normal stresses predicted with the ordinary finite element meshes I, III and V are plotted in Figure 7. We observe that the normal stress oscillates spuriously around the singularity; the oscillations on the wall are of smaller frequency and greater amplitude than those on the slip 


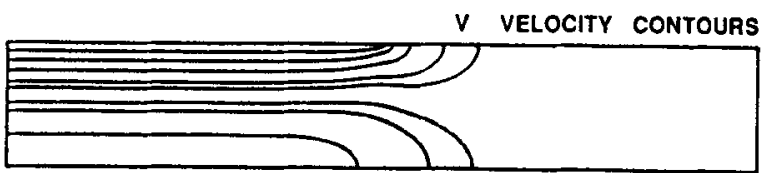

(a)

W VELOCITY CONTOURS

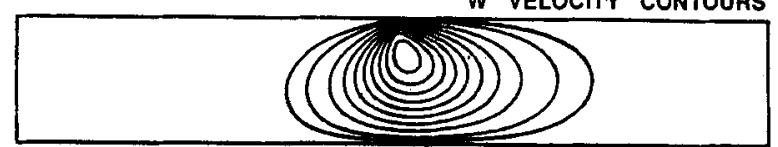

(b)

PRESSURE CONTOURS

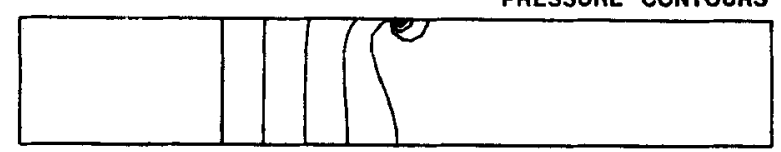

(c)

STREAMLINES

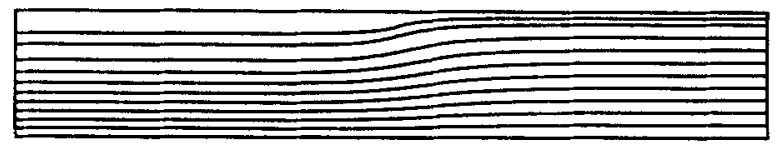

(d)

Figure 5. Solution of the stick-slip problem: (a) $y$-velocity; (b) $z$-velocity; (c) pressure; (d) streamlines

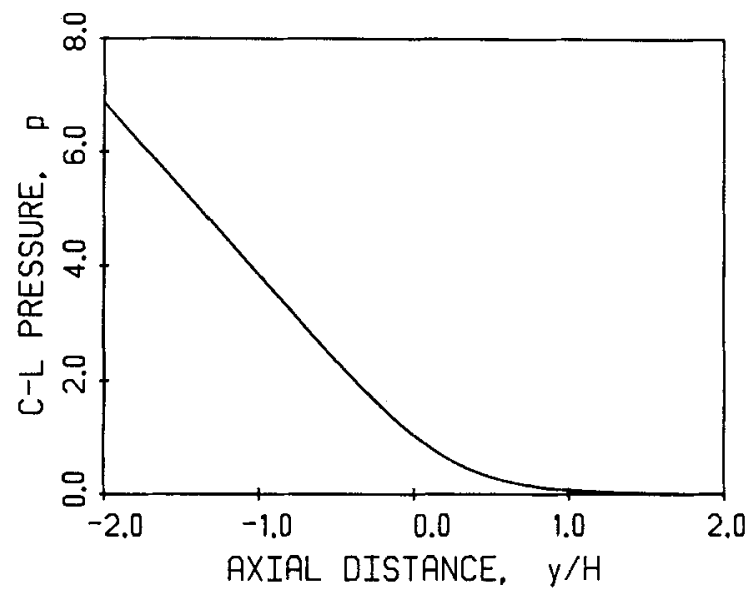

Figure 6. Predicted centreline pressure with singular and ordinary elements

surface. As we refine the mesh, the oscillations move towards the singular point and their amplitude increases.

By using singular finite elements, we practically eliminate these oscillations. The normal stress obtained with the coarsest singular mesh is smooth and agrees well with the analytical solution (Figure 8). Note that the coarsest mesh gives virtually the same normal stress results as the finest mesh, indicating that relatively coarse meshes may be used with singular finite elements. However, some oscillations of very small amplitude still occur very close to the singularity with the finer meshes. These oscillations are not restricted to the singular elements but also appear in the 


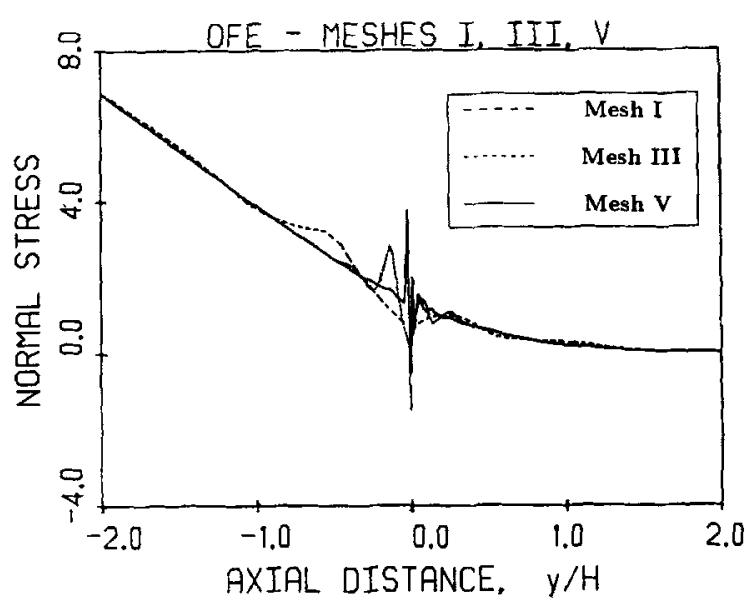

Figure 7. Predicted normal stress at $z=1$ with ordinary elements for planar stick-slip problem: - - , mesh I; .$- \cdot$, mesh III; - - mesh V

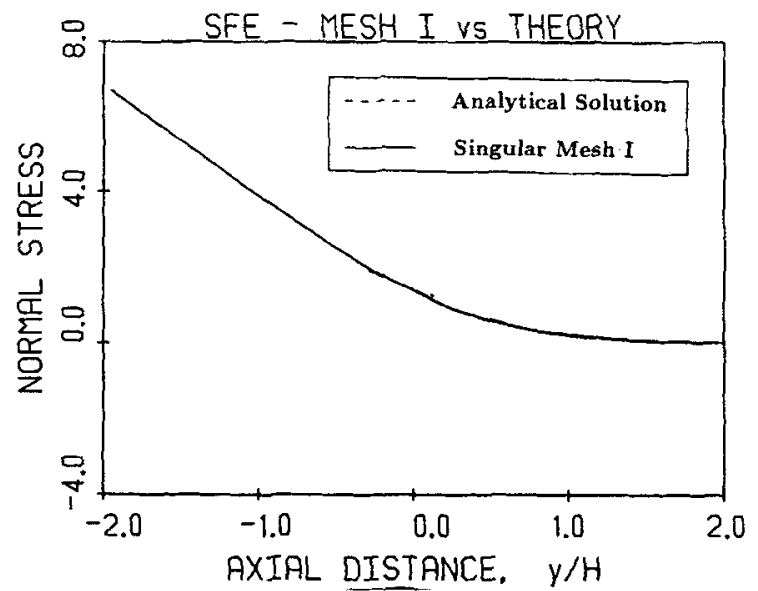

Figure 8. Normal stresses at $z=1: \_$, singular elements, mesh I; - - , theory

surrounding ordinary elements; this may be due to the fact that the pressure and viscous stress grow large with opposite signs, which gives rise to a numerical error.

No matter what the cause of the oscillations is, the main disadvantage of the embodied singularity elements is apparent: by refining the mesh, we reduce the size of the singular elements over which the singularity is given special attention. Nevertheless, this problem may be partially resolved by restructuring the mesh so that the size of the singular elements is fixed and independent of mesh refinement (e.g. by using more ordinary transition elements or by using triangular instead of rectangular ordinary elements).

The normal stress results were used to choose the order and the number of the singular elements and to check whether ordinary transition elements are necessary around the singular elements. In addition to the aforementioned pattern with eight 13-node singular elements and eight transition elements, three alternative mesh patterns were also examined: (i) eight 7-node singular elements 
with eight ordinary transition elements, (ii) four 13-node singular elements with four ordinary transition elements and (iii) eight 13-node singular elements with no ordinary transition elements. All the examined mesh patterns are shown in Figure 9. The predicted normal stresses were better than those obtained with ordinary elements in all cases. However, the results with the alternative mesh patterns examined were characterized by some oscillations, which disappear with mesh refinement. The most severe oscillations occur with the 7-node singular elements (Figure 10). This

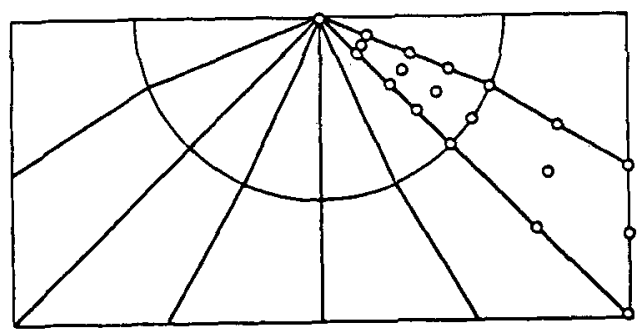

(a)

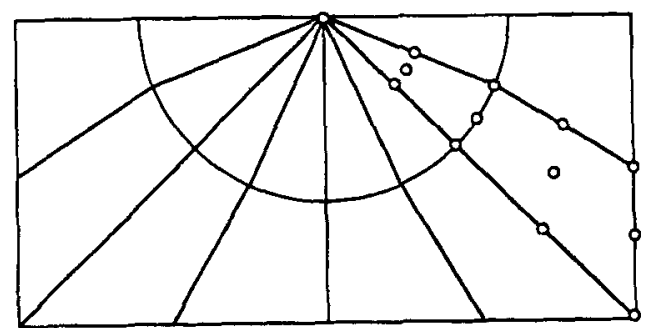

(c)

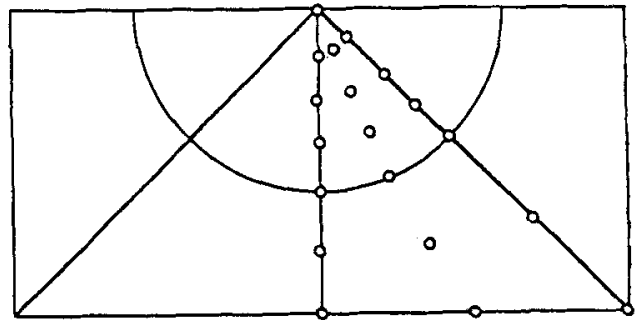

(b)

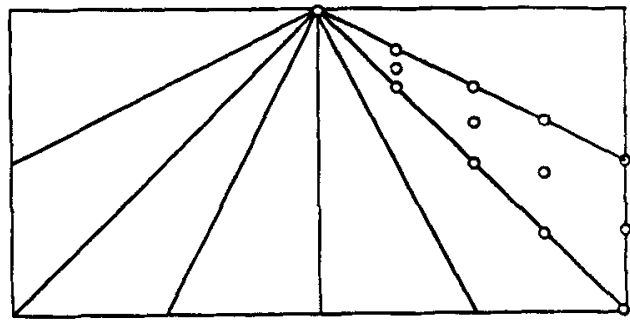

(d)

Figure 9. Examined mesh patterns around the singular point

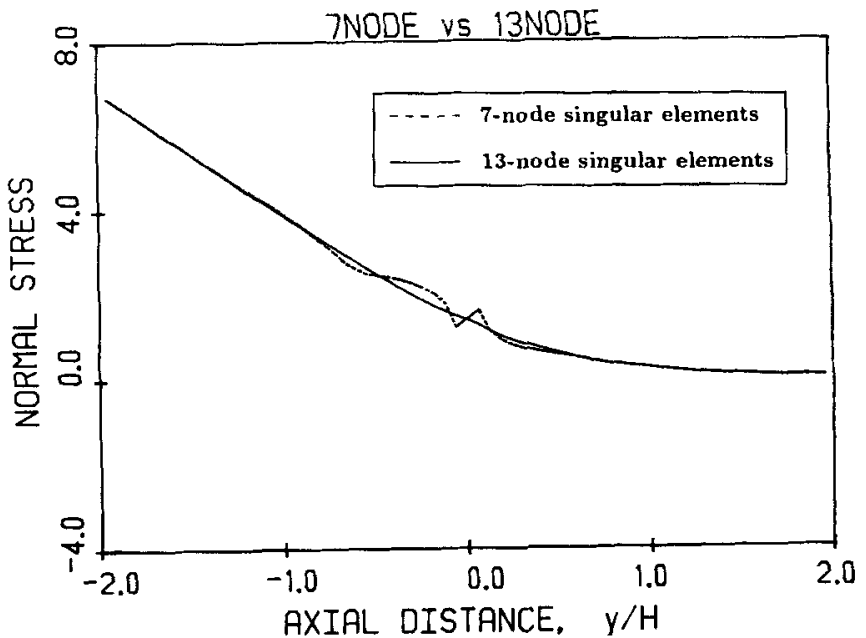

Figure 10. Computed normal stresses with 7-node $(--)$ and 13-node (-) singular elements (mesh I) 
is due not only to the lower order of the element but also to the fact that some terms of the basis functions become zero in accordance with the analytical solution. Consequently, only one term survives to express the radial dependence of the normal stress. 13-node elements overcome this difficulty. The mesh pattern with eight 13-node singular elements and eight ordinary transition elements appeared to give satisfactory results and adequately captured the $\theta$ dependence of the local solution. After some preliminary tests, the radius of the singular elements was taken to be 1.2 times the size of the original ordinary square.

To assure that the improved solution is due to the singular basis functions and not to the different shapes and mesh patterns used with the singular elements, we solved the problem by replacing the 13-node singular elements with 13-node ordinary elements. Comparison of the computed normal stresses (Figure 11) indicates that the singular basis functions are important for an improved solution.

As with the use of singular basis functions, ${ }^{8}$ the condition number of the stiffness matrix becomes larger if singular elements are used. Table I summarizes how the condition number changes with the mesh and type of element. The condition number, computed using the LINPACK subroutine DGBCO, was sufficiently small for all our double-precision computations.

The second quantity we examined was the velocity on the slip surface. The computed nodal slip surface velocities from ordinary mesh IV and singular mesh II are shown in Figure 12, along with the asymptotic solution. The singular finite element values agree well with the analytical solution;

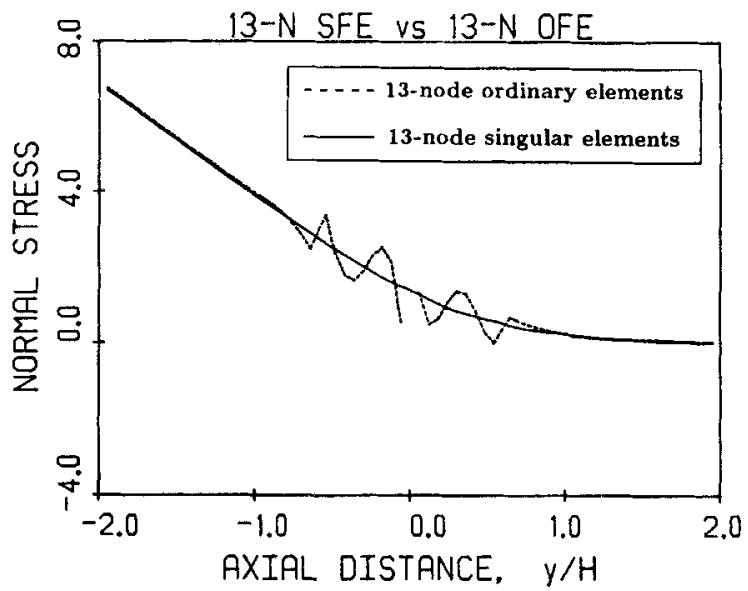

Figure 11. Computed normal stresses with 13-node ordinary (- -) and singular (- ) elements (mesh I)

Table I. Inverse condition number of the stiffness matrix for various elements and meshes

\begin{tabular}{llc}
\hline Mesh & Element & 1 cond $(A)$ \\
\hline I & Ordinary 9-node & $3.234 \times 10^{-4}$ \\
II & Ordinary 9-node & $1.015 \times 10^{-4}$ \\
III & Ordinary 9-node & $4.009 \times 10^{-5}$ \\
I & Singular 7-node & $2.394 \times 10^{-5}$ \\
II & Singular 7-node & $7.236 \times 10^{-6}$ \\
I & Singular 13-node & $2.456 \times 10^{-7}$ \\
II & Singular 13-node & $7.505 \times 10^{-8}$ \\
\hline
\end{tabular}




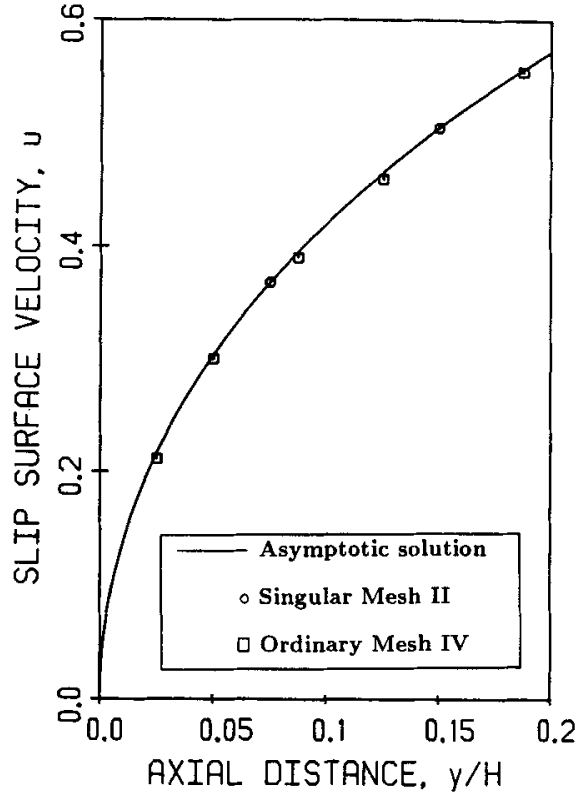

Figure 12. Comparison of predicted slip surface velocities near the singularity: ——, asymptotic solution; $O$, singular mesh II; $\square$, ordinary mesh IV

Table II. Computed singularity expansion coefficients

\begin{tabular}{lccr}
\hline Method & $\alpha_{1 / 2}$ & $\alpha_{3 / 2}$ & \multicolumn{1}{c}{$\alpha_{5 / 2}$} \\
\hline Ordinary elements $_{\text {Singular elements }}$ & 0.67170 & 0.19812 & -0.02297 \\
Ingham and Kelmanson $^{10}$ & 0.69173 & 0.27168 & 0.05013 \\
Analytical solution $^{10}$ & 0.69108 & 0.26435 & 0.04962 \\
\hline
\end{tabular}

the ordinary element results differ slightly and converge to the analytical solution with mesh refinement. Again, the coarse singular element meshes give more accurate results than refined ordinary meshes.

For another comparison with the analytical solution, we estimated the first expansion coefficients using a least-squares fit of equation (5) to the eight nodal slip surface velocities closest to the singularity. Estimates of the first three expansion coefficients are given by Ingham and Kelmanson ${ }^{10}$ who used a singular boundary integral method to solve the planar stick-slip problem. The estimated parameters for mesh III are listed in Table II along with the extrapolated values given in Reference 10 . The agreement with the analytical solution is satisfactory despite the fact that the least-squares fit was not rigorous with regard to the number and the weight of the nodal points.

\section{CONCLUSIONS}

Singular finite elements have been developed for the stick-slip problem in order to improve the solution in the neighbourhood of the singularity. These elements are similar in principle to the 
crack tip elements used in fracture mechanics; however, in fluid flow problems the pressure, a primary unknown, is singular. Compared to ordinary finite elements, the singular elements give more accurate results for relatively coarse meshes. Good approximations to the leading singular coefficients have also been obtained. The method can be readily applied to other problems in fluid mechanics for which the radial form of the singularity can be obtained by a local analysis. As mentioned in Section 1, the method is not restricted to creeping and Newtonian flows, but is also applicable to non-zero Reynolds number and non-Newtonian flows provided that the encountered stresses are integrable.

From some preliminary results, ${ }^{34}$ it appears that the removal of the pressure node from the singular point plays a crucial role in the improvement of the solution. Singular finite elements, with an approximate singularity exponent independent of the free surface shape and location, can be used to study singular free surface problems. They can also be used for problems with nonintegrable singularities by approximating the singular quantities by $r^{-1+\varepsilon}$, where $0<\varepsilon \ll 1$. These issues are currently under investigation.

\section{ACKNOWLEDGEMENTS}

This work was partially supported by the National Science Foundation under contract MSM8504456 and the Office of the Vice President for Research, University of Michigan.

\section{APPENDIX: MAPPING FOR SINGULAR ELEMENTS}

As in Tracey, ${ }^{9}$ we demonstrate that the $s$-co-ordinate for the element shown in Figure 2 is approximately mapped to the radial direction, and the $t$-co-ordinate is mapped to the circumferential angle. Suppose that the global radius of the element is given by $R$ and the total global angle subtended by the element is $\beta$. By directly applying the standard polynomial shape functions for an element, we find that

$$
y=\frac{R}{2}(1+s)\left(\frac{t}{2}(1+t)+\cos \frac{\beta}{2}\left(1-t^{2}\right)-\cos \beta \frac{t}{2}(1-t)\right) \sim \frac{R}{2}(1+s)
$$

and

$$
z=\frac{R}{2}(1+s)\left(-\sin \frac{\beta}{2}\left(1-t^{2}\right)+\sin \beta \frac{t}{2}(1-t)\right) \sim-\frac{R \beta}{4}(1+s)(1-t),
$$

where the simplifications use small-angle approximations. Then the determinant of the Jacobian matrix is

$$
|\mathbf{J}|=\frac{R^{2}}{8} \beta(1+s)
$$

i.e. the differential area of the singular element is $r \mathrm{~d} r \mathrm{~d} \theta=|\mathbf{J}| \mathrm{d} s \mathrm{~d} t$.

\section{REFERENCES}

1. R. E. Nickell, R. I. Tanner and B. Caswell, 'The solution of viscous incompressible jet and free-surface flows using finite element methods', J. Fluid Mech., 65, 189-205 (1974).

2. K.J. Ruschak, 'A method for incorporating free boundaries with surface tension in finite element fluid-flow simulators', Int. j. numer. methods eng., 15, 639-647 (1980).

3. B. J. Omodei, 'Computer solutions of a plane Newtonian jet with surface tension', Comput. Fluids, 7, 79-96 (1979).

4. G. C. Georgiou, T. C. Papanastasiou and J. O. Wilkes, 'Laminar Newtonian jets at high Reynolds and high surface tension', AIChE J., 34, 1559-1562 (1988). 
5. P. Andre and J.-R. Clermont, "Numerical simulation of the die swell problem of a Newtonian fluid by using the concept of stream function and a local analysis of the singularity at the corner', J. Non-Newtonian Fluid Mech., 23, 335-357 (1987).

6. W. J. Silliman and L. E. Scriven, 'Separating flow near a static contact line. Slip at a wall and shape of a free surface', J. Comput. Phys., 34, 287-313 (1980).

7. J. R. Whiteman, 'Numerical solution of steady state diffusion problems containing singularities', in R. H. Gallagher, J. T. Oden, C. Taylor and O. C. Zienkiewicz (eds), Finite Elements in Fluids, Vol. 2, Wiley 1985.

8. G. Strang and G. J. Fix, An Analysis of the Finite Element Method, Prentice-Hall, Englewood Cliffs, NJ, 1973.

9. D. M. Tracey, 'Finite elements for determination of crack tip elastic stress intensity factors', Eng. Fract. Mech., 3, 255-265 (1971).

10. D. B. Ingham and M. A. Kelmanson, Boundary Integral Equation Analyses of Singular, Potential, and Biharmonic Problems, Springer-Verlag, Berlin, 1984, pp. 21-51.

11. M. Kermode, A. McKerrell and L. M. Delves, 'The calculation of singular coefficients', Comput. Methods Appl. Mech. Eng., 50, 205-215 (1985).

12. N. Y. Lee, W. W. Schultz and J. P. Boyd, 'Spectral solutions for flows with corner singularities', APS Division of Fluid Mechanics 40th Annual Meeting, Eugene, OR, 1987.

13. L. S. D. Morley, 'Finite element solution of boundary-value problems with non-removable singularities', Phil. Trans. R. Soc. Lond. A, 275, 463-488 (1973).

14. D. M. Tracey and T. S. Cook, 'Analysis of power type singularities using finite elements', Int. j. numer. methods eng., 11, $1225-1233$ (1977).

15. H. G. Askar, 'Special elements for point singularities', Comput. Methods Appl. Mech. Eng., 63, 271-280 (1987).

16. G. C. Sih (ed.), Methods of Fracture I. Methods of Analysis and Solutions of Crack Problems, Nordofl, Leyden, 1973.

17. R. D. Henshell and K. G. Shaw, 'Crack tip elements are unnecessary', Int. j. numer. methods eng., 9, $495-507$ (1975).

18. R. Wait, 'Singular isoparametric finite elements', J. Inst. Math. Applic., 20, 133-141 (1977).

19. R. S. Barsoum, 'Triangular quarter-point elements as elastic and perfectly-plastic crack-tip elements', Int, $j$. numer. methods eng., 11, 85-98 (1977).

20. H. Gallagher, 'Finite element analysis for crack tip problems', in Proc. Symp. on Finite Element Method, Science Press, Beijing, China, 1982, pp. 102-149.

21. S. Richardson, 'A 'stick-slip' problem related to the motion of a free jet at low Reynolds numbers', Proc. Camb. Phil. Soc., 67, 477-489 (1970).

22. L. D. Sturges, Ph.D. Thesis, University of Minnesota, 1977.

23. D. H. Michael, 'The separation of a viscous liquid at a straight edge', Mathematika, 5, 82-84 (1958).

24. H. K. Moffat, 'Viscous and resistive eddies near a sharp corner', J. Fluid Mech., 18, 1-18 (1964).

25. W. W. Schultz and C. Gervasio, 'An analysis of the singularity in the die swell problem', 10th Int. Congr. on Rheology, Sydney, 1988.

26. R. R. Huilgol and R. I. Tanner, 'The separation of a second-order fluid at a straight edge', J. Non-Newtonian Fluid Mech., 2, 89-96 (1977).

27. M. R. Apelian, R. C. Armstrong and R. A. Brown, 'Impact of the constitutive equation and singularity on the calculation of stick-slip flow: the modified upper-convected Maxwell model (MUCM)', J. Non-Newtonian Fluid Mech., 27, 299-321 (1988).

28. T. J. R. Hughes and J. E. Akin, 'Techniques for developing "special" finite element shape functions with particular reference to singularities', Int. j. numer. methods eng., 15, 733-751 (1980).

29. K. J. Bathe, Finite Element Procedures in Engineering Analysis, Prentice-Hall, Englewood Cliffs, NJ, 1982.

30. A. J. Baker, Finite Element Computational Fluid Mechanics, McGraw-Hill, New York, 1983.

31. M. Okabe, 'Fundamental theory of the semi-radial singularity mapping with applications to fracture mechanics', Comput. Methods Appl. Mech. Eng., 26, 53-73 (1981).

32. J. S. Solecki and J. L. Swedlow, 'On quadrature and singular finite elements', Int. j. numer. methods eng., 20, 395-408 (1984).

33. M. Stern, 'Families of consistent conforming elements with singular derivative fields', Int. $j$. numer. methods eng., 14, 409-421 (1979).

34. G. C. Georgiou, W. W. Schultz and L. Olson, 'Singular finite elements for fluid flow problems', APS Division of Fluid Mechanics 41st Annual Meeting, Buffalo, NY, 1988. 